\title{
DERIVATIONS IN PRIME RINGS ${ }^{1}$
}

\author{
EDWARD C. POSNER
}

We prove two theorems that are easily conjectured, namely: (1) In a prime ring of characteristics not 2 , if the iterate of two derivations is a derivation, then one of them is zero; (2) If $d$ is a derivation of a prime ring such that, for all elements $a$ of the ring, $a d(a)-d(a) a$ is central, then either the ring is commutative or $d$ is zero.

Definition. $A$ ring $R$ is called prime if and only if $x a y=0$ for all $a \in R$ implies $x=0$ or $y=0$.

From this definition it follows that no nonzero element of the centroid has nonzero kernel, so that we can divide by the prime $p$, unless $p x=0$ for all $x$ in $R$, in which case we call $R$ of characteristic $p$.

A known result that will be often used throughout this paper is given in

Lemma 1. Let $d$ be a derivation of a prime ring $R$ and a be an element of $R$. If $a d(x)=0$ for all $x \in R$, then either $a=0$ or $d$ is zero.

Proof: In $a d(x)=0$ for all $x \in R$, replace $x$ by $x y$. Then

$$
a d(x y)=0=a d(x) y+a x d(y)=\operatorname{axd}(y)=0
$$

for all $x, y \in R$. If $d$ is not zero, that is, if $d(y) \neq 0$ for some $y \in R$, then, by the definition of a prime ring, $a=0$.

The following lemma may have some independent interest.

Lemma 2. Let $R$ be a prime ring, and let $p, q, r$ be elements of $R$ such that paqar $=0$ for all $a$ in $R$. Then one, at least, of $p, q, r$ is zero.

Proof. In paqar $=0$, replace $a$ by $a+b$; using paqar $=p b q b r=0$, we find paqbr $+p b q a r=0$, for all $a, b$ in $R$. If now $p a=0$, then, for all $b$ in $R$, pbqar $=0$, so that $p=0$, or else $q a r=0$. But if $p a=0$, then pat $=0$ for all $t \in R$, so that $p=0$ or $q a t r=0$ for all $t$ in $R$; again $r=0$, or else $q a=0$. So $p=0$ or $r=0$ or $q a$ is zero whenever $p a$ is zero; replace $a$ by aqar; since $p$ (aqar) $=0$ for all $a \in R$, we see that $p=0$ or $r=0$ or qaqar $=0$ for all $a \in R$. Similarly, $p=0$ or $r=0$ or $q a q a q=0$ for all $a \in R$. Assuming therefore that $p \neq 0, r \neq 0$, replace $a$ by $a+b$ in $q a q a q=0$ to find as before that $q a q b q+q b q a q=0$. In this equation, replace $b$ by $a q b$ to find 1957.

Presented to the Society February 23, 1957; received by the editors February 7,

1 This paper was sponsored in part by the Office of Ordnance Research, United States Army, under contract DA-11-022-ORD-1571. 
$(q a q a q) b q+q a q b q a q=0,(q a q) b(q a q)=0$, for all $b \in R$, for all $a \in R$. So $q a q=0$ for all $a \in R, q=0$ if $p \neq 0, r \neq 0$.

TheOREM 1. Let $R$ be a prime ring of characteristic not 2 and $d_{1}, d_{2}$ derivations of $R$ such that the iterate $d_{1} d_{2}$ is also a derivation; then one at least of $d_{1}, d_{2}$ is zero.

Proof. $d_{1} d_{2}$ is a derivation, so

$$
d_{1} d_{2}(a b)=d_{1} d_{2}(a) b+a d_{1} d_{2}(b) .
$$

However, $d_{1}, d_{2}$ are each derivations so

$$
\begin{aligned}
d_{1} d_{2}(a b) & =d_{1}\left(d_{2}(a b)\right)=d_{1}\left(d_{2}(a) b+a d_{2}(b)\right) \\
& =d_{1} d_{2}(a) b+d_{2}(a) d_{1}(b)+d_{1}(a) d_{2}(b)+a d_{1} d_{2}(b) .
\end{aligned}
$$

But $d_{1} d_{2}(a b)=d_{1} d_{2}(a) b+a d_{1} d_{2}(b)$, so

$$
d_{2}(a) d_{1}(b)+d_{1}(a) d_{2}(b)=0 \quad \text { for all } a, b \in R .
$$

Replace $a$ by $a d_{1}(c)$ in (1).

$$
d_{2}\left(a d_{1}(c)\right) d_{1}(b)+d_{1}\left(a d_{1}(c)\right) d_{2}(b)=0
$$

for all $a, b, c \in R$.

$$
d_{2}(a) d_{1}(c) d_{1}(b)+a d_{2} d_{1}(c) d_{1}(b)+d_{1}(a) d_{1}(c) d_{2}(b)+a d_{1}^{2}(c) d_{2}(b)=0 .
$$

Now $a\left(d_{2}\left(d_{1}(c)\right) d_{1}(b)+d_{1}\left(d_{1}(c)\right) d_{2}(b)\right)=0, \quad$ since $\quad d_{2}\left(d_{1}(c)\right) d_{1}(b)+d_{1}$ $\cdot\left(d_{1}(c)\right) d_{2}(b)=0$, which is merely equation (1) with $a$ replaced by $d_{1}(c)$. We are left, then, with

$$
d_{2}(a) d_{1}(c) d_{1}(b)+d_{1}(a) d_{1}(c) d_{2}(b)=0 \quad \text { for all } a, b, c \in R .
$$

But $d_{1}(c) d_{2}(b)=-d_{2}(c) d_{1}(b)$ by (1) with $c$ replacing $a$. Then (2) becomes $d_{2}(a) d_{1}(c) d_{1}(b)-d_{1}(a) d_{2}(c) d_{1}(b)=0$; factoring out $d_{1}(b)$ on the right, we have $\left(d_{2}(a) d_{1}(c)-d_{1}(a) d_{2}(c)\right) d_{1}(b)=0$ for all $b \in R$, for all $a$, $c \in R$. Lemma 1 is just what we need to tell us that $d_{2}(a) d_{1}(c)$ $-d_{1}(a) d_{2}(c)=0$ for all $a, c \in R$, unless $d_{1}$ is zero. But (1) with $c$ replacing $b$ tells us that instead $d_{2}(a) d_{1}(c)+d_{1}(a) d_{2}(c)=0$ for all $a, c \in R$. Adding these last two equations, we find that $2 d_{2}(a) d_{1}(c)=0$, $d_{2}(a) d_{1}(c)=0$, (since $R$ is not of characteristic 2), for all $a, c \in R$, or else $d_{1}$ is zero. Using Lemma 1 again with $d_{2}(a)$ replacing $a$, we find that $d_{1}$ is zero or else $d_{2}(a)=0$ for all $a \in R$, i.e. $d_{1}=0$ or $d_{2}=0$.

In order to prove Theorem 2, we find it necessary to prove the following lemma.

Lemma 3. Let $R$ be a prime ring, and $d$ a derivation of $R$ such that $a d(a)-d(a) a=0$ for all $a \in R$. Then $R$ is commutative, or $d$ is zero. 
Proof. $(a+b) d(a+b)-(d(a+b))(a+b)=0$ for all $a, b \in R$; subtracting $a d(a)-d(a) a+b d(b)-d(b) b=0$ from this, we arrive at $a d(b)+b d(a)-d(a) b-d(b) a=0$ for all $a, b \in R$. Write this as

$$
a d(b)-d(a) b=d(b) a-b d(a) .
$$

Add to this $a d(b)+d(a) b=d(a b)$ to find

$$
2 a d(b)=d(b) a-b d(a)+d(a b) \quad \text { for all } a, b \in R .
$$

In (3), replace $b$ by $a x$

or

$$
2 a d(a x)=d(a x) a-a x d(a)+d\left(a^{2} x\right),
$$

$$
2 a d(a) x+2 a^{2} d(x)=d(a) x a+a d(x) a-a x d(a)+2 a d(a) x+a^{2} d(x),
$$
since $d\left(a^{2}\right)=2 a d(a)$; or

$$
a^{2} d(x)=d(a) x a+a d(x) a-\operatorname{axd}(a) \quad \text { for all } a, x \in R .
$$

In (3), replace $b$ by $x a$, and find similarly

$$
d(x) a^{2}=a d(x) a+\operatorname{axd}(a)-d(a) x a, \quad \text { for all } a, x \in R .
$$

Add (4) and (5).

$$
a^{2} d(x)+d(x) a^{2}=2 a d(x) a \quad \text { for all } a, x \in R,
$$

or

$$
a(d(x) a-a d(x))=(d(x) a-a d(x)) a \quad \text { for all } a, x \in R .
$$

Replace in (7) $a$ by $a+d(x)$; we find that $d(x)$ commutes with $d(x) a-a d(x)$, for all $a \in R$, for all $x$ in $R$; this says that the square of the inner derivation by $x$ is zero, for all $x \in R$. Let $R$ not be of characteristic 2. Then Theorem 1 says that $d(x)$ is central, for all $x$ in $R$; let $a$ be an element of $R$, and $A$ denote inner derivation by $a . a d(x)$ $=d(x) a$, or $A d(x)=0$ for all $x \in R$. Theorem 1 again shows that $d=0$ or, if not, then $A$ is zero, every $a$ in $R$ is central, $R$ is commutative. But if $R$ is of characteristic 2, (6) says that for all $x \in R, d(x)$ commutes with all squares of elements of $R$. Let $R$ be a prime ring of characteristic 2, and let $e \in R$ commute with $a^{2}$, for all $a \in R$.

$$
a^{2} e=e a^{2}
$$

for all $a \in R$.

Replace $a$ by $a+b$ and use $e a^{2}=a^{2} e, e b^{2}=b^{2} e$.

$$
(a b+b a) e=e(a b+b a) \quad \text { for all } a, b \in R .
$$

In (9), replace $b$ by $a e$ and commute $e$ and $a^{2}$; then $a^{2} e^{2}+a e a e=e a^{2} e$ $+e a e a ; a^{2} e^{2}=e a^{2} e$, so 


$$
a e a e=e a e a
$$

for all $a \in R$.

In (9), replace $b$ by $e$; then $a e^{2}+e a e=e a e+e^{2} a$,

$$
e^{2} \text { is in the center of } R \text {. }
$$

Consider $(a e+e a)^{2}=a e a e+e a e a+a e^{2} a+e a^{2} e$. But $a e a e+e a e a=0$ by (10), $a e^{2} a+e a^{2} e=e^{2} a^{2}+e^{2} a^{2}=0$ by (11) and (8). We have

$$
(a e+e a)^{2}=0 \quad \text { for all } a \in R .
$$

Let $x, y$ now be elements of $R$ with $x y=0$. By (9), $(x y+y x) e$ $=e(x y+y x)$, so

$$
x y=0 \text { implies } y x e=e y x \text {. }
$$

Now $x^{2} y=0$, so (13) becomes also $y x^{2} e=e y x^{2} ; y x^{2} e=y e x^{2}$ since $e$ commutes with all squares. Thus

$$
x y=0 \text { implies }(y e+e y) x^{2}=0 .
$$

But ( $a x) y=0$ for all $a \in R$; then we can replace $x$ by $a x$ in (14), to obtain $(y e+e y) a x a x=0$ for all $a \in R$, whenever $x y=0$. Lemma 2 now says $x=0$ or $y e+e y=0$; in fact, since $x(y v)=0$ for all $v \in R$, Lemma 2 even says $x=0$ or $y v e+(e y) v=0$ for all $v \in R$. Since $y e=e y$ if $x \neq 0$, then $x=0$ or $y v e+y e v=0$ for all $v \in R, y(v e+e v)=0$ for all $v \in R$. Lemma 1 applied to the inner derivation by $e$ shows that either $x=0, y=0$, or $e$ is central. But by (12) $(a e+e a)(a e+e a)=0$, for all $a \in R$; putting $x=a e+e a, y=a e+e a$, we find that for all $a \in R$, $a e+e a=0$, or $e$ is central. That is, for all $a \in R, a e+e a=0, e$ is central if $e$ commutes with all squares in $R$.

For all $x \in R$, then, $d(x)$ commutes with all squares in $R, d(x)$ is central for all $x \in R$. Let $d(b)=0$; for all $a \in R, d(a b)=d(a) b+a d(b)$ $=d(a) b ; d(a b)$ is central, so $d(a) b$ is central for all $a$ in $R$ if $d(b)=0$. Now if $d$ is not zero, so that $d(a) \neq 0$ for some $a \in R$, we have $d(a) b x$ $=x d(a) b ; d(a)$ is central so $x d(a) b=d(a) x b$, whence $d(a)(b x+x b)=0$ for all $x \in R$, if $d(b)=0$. But as previously remarked, no nonzero element of the centroid of $R$ has nonzero kernel; since we are assuming $d(a) \neq 0$, and since $d(a)$ is central, we have proved that $b$ is central whenever $d(b)=0$. But for all $c \in R, d\left(c^{2}\right)=d(c) c+c d(c)=2 d(c) c=0$, so $c^{2}$ commutes with all $x$ in $R$, for all $c \in R$. Referring back to the conclusion of the previous paragraph with $x$ for $e$ shows $x$ central for all $x \in R$, if $d$ is not the zero derivation.

The following lemma may also be of independent interest.

Lemma $4 .{ }^{2}$ Let $A$ be a Lie ring, $I$ an ideal of $A, d$ an element of $A$ such

${ }^{2}$ An oral communication from Professor Kaplansky. 
that $d x \cdot x=0$ for all $x \in I$. Then for all $a \in R,(d a \cdot x) x=0$ for all $x \in I$ (i.e. the set of $d$ satisfying $d x \cdot x=0$ for all $x \in I$ is an ideal of $A$ ).

Proof. Let $R_{a}$ denote right multiplication by $a$. We want to prove $d\left(R_{a} R_{x}^{2}\right)=0$ for all $a \in A, x \in I$. The Jacobi identity for a Lie ring may be written as $R_{a x}=R_{a} R_{x}-R_{x} R_{a}$. Furthermore, since $I$ is an ideal, it contains $a x$, and $x+a x$, for all $a \in A$, so that $(d \cdot a x) a x=0,(d(x+a x))$ $\cdot(x+a x)=0$ for all $a \in A$. From these two equations, and from $d x \cdot x=0$, we get $d x \cdot a x+(d \cdot a x) \cdot x=0$ for all $a \in A, x \in I$, or, in the other notation, $d\left(R_{x} R_{a x}+R_{a x} R_{x}\right)=0$. But from

$$
\begin{aligned}
d\left(R_{x} R_{a x}+\right. & \left.R_{a x} R_{x}\right)=d\left(R_{x}\left(R_{a} R_{x}-R_{x} R_{a}\right)+\left(R_{a} R_{x}-R_{x} R_{a}\right) R_{x}\right) \\
& =d\left(R_{x} R_{a} R_{x}-R_{x}^{2} R_{a}+R_{a} R_{x}^{2}-R_{x} R_{a} R_{x}\right)=d\left(R_{a} R_{x}^{2}-R_{x}^{2} R_{a}\right),
\end{aligned}
$$

$d\left(R_{a} R_{x}^{2}-R_{x}^{2} R_{a}\right)=0$ for all $a \in A, x \in I$. By hypothesis, $d\left(R_{x}^{2}\right)=0$, so that $d\left(R_{a} R_{x}^{2}\right)=0$ for all $a \in A, x \in I$. This is exactly what we had to prove.

We are now ready for Theorem 2 .

THEOREM 2. Let $R$ be a prime ring and $d$ a derivation of $R$ such that, for all $a \in R, a d(a)-d(a) a$ is in the center of $R$. Then, if $d$ is not the zero derivation, $R$ is commutative.

Proof. Let $A$ be the Lie ring of derivations of $R$ and $I$ the ideal of $A$ consisting of inner derivations. Let, for $a \in R, I_{a}$ denote inner derivation by $a$. Let $\left[d_{1}, d_{2}\right]$ for $d_{1}, d_{2} \in A$ denote the (commutator) product of derivations in $A$. We are assuming $\left[\left(d, I_{a}\right), I_{a}\right]=0$. By the preceding lemma, for all $x \in R$, that is, for all $I_{x} \in I$, $\left[\left[\left[d, I_{x}\right] I_{a}\right] I_{a}\right]=0$ for all $a \in R$. That is, $a(a d(x)-d(x) a)-(\operatorname{ad}(x)$ $-d(x) a) a$ is central for all $x, a \in R$,

$$
a^{2} d(x)+d(x) a^{2}-2 a d(x) a \text { is central for all } x, a \in R .
$$

Commute (15) with $a$.

$$
3 a d(x) a^{2}+a^{3} d(x)=3 a^{2} d(x) a+d(x) a^{3} .
$$

Suppose $R$ is of characteristic 3. Then for all $a \in R, I_{a}^{3} d=0$. Theorem 1 says that $d$ is zero, or else every $a^{3}$ is in the center of $R$; if this is the case, then for all $a, b \in R,(a+b)^{3}-a^{3}-b^{3}=a^{2} b+a b a+b a^{2}+b^{2} a+b a b$ $+a b^{2}$ is central; replace $a$ by $-a$ to find $a^{2} b+a b a+b a^{2}-\left(b^{2} a+b a b+a b^{2}\right)$ central for all $a, b \in R$; adding these last two and dividing by 2 , we see that $a^{2} b+a b a+b a^{2}$ is central, for all $a, b \in R$. Replace $b$ by $a b$ : $a^{3} b+a^{2} b a+a b a^{2}=a\left(a^{2} b+a b a+b a^{2}\right)$ is central; if $a^{2} b+a b a+b a^{2}$ is not zero, given $a$, for some $b$, then, since it is central, we can divide by it, whence $a$ would be central. So assume that $R$ has the property that 
for all $a, b \in R, a^{2} b+a b a+b a^{2}=0$. This reads, since $R$ is of characteristic 3, as $a(a b-b a)-(a b-b a) a=0$ for all $b \in R, I_{a}^{2}=0$; by Theorem $1, a$ is central, $R$ is commutative.

Suppose now that $R$ is of characteristic different from 3 . Write $d(x)=x^{\prime}$. In (16), replace $x$ by $a: 3 a a^{\prime} a^{2}+a^{3} a^{\prime}-3 a^{2} a^{\prime} a-a^{\prime} a^{3}=0$, or $a^{3} a^{\prime}-a^{\prime} a^{3}=3 a^{2} a^{\prime} a-3 a a^{\prime} a^{2}=3 a\left(a a^{\prime}-a^{\prime} a\right) a$. Since $a a^{\prime}-a^{\prime} a$ is central by the hypothesis of this theorem, we find

$$
a^{3} a^{\prime}-a^{\prime} a^{3}=3\left(a a^{\prime}-a^{\prime} a\right) a^{2}, \quad \text { for all } a \in R .
$$

Furthermore, $\left(a a^{\prime}-a^{\prime} a\right) a=a a^{\prime} a-a^{\prime} a^{2}$. But $\left(a a^{\prime}-a^{\prime} a\right) a=a\left(a a^{\prime}-a^{\prime} a\right)$ $=a^{2} a^{\prime}-a a^{\prime} a$; adding these last two, we obtain

$$
2\left(a a^{\prime}-a^{\prime} a\right) a=a^{2} a^{\prime}-a^{\prime} a^{2} .
$$

In (16), replace $x$ by $a x^{\prime}$.

$$
\begin{gathered}
3 a^{2} x^{\prime \prime} a^{2}+a^{4} x^{\prime \prime}-3 a^{3} x^{\prime \prime} a-a x^{\prime \prime} a^{3}+3 a a^{\prime} x^{\prime} a^{2}+a^{3} a^{\prime} x^{\prime} \\
-3 a^{2} a^{\prime} x^{\prime} a-a^{\prime} x^{\prime} a^{3}=0 .
\end{gathered}
$$

However,

$$
\begin{aligned}
3 a^{2} x^{\prime \prime} a^{2}+a^{4} x^{\prime \prime}-3 a^{3} x^{\prime \prime} a- & a x^{\prime \prime} a^{3} \\
& =a\left(3 a x^{\prime \prime} a^{2}+a^{3} x^{\prime \prime}-3 a^{2} x^{\prime \prime} a-x^{\prime \prime} a^{3}\right)=0,
\end{aligned}
$$

as is seeen from (16) by replacing $x$ by $x^{\prime}$. So

$$
3 a a^{\prime} x^{\prime} a^{2}+a^{3} a^{\prime} x^{\prime}-3 a^{2} a^{\prime} x^{\prime} a-a^{\prime} x^{\prime} a^{3}=0 \quad \text { for all } x, a \in R .
$$

Multiply (16) on the left by $a^{\prime}$.

$$
3 a^{\prime} a x^{\prime} a^{2}+a^{\prime} a^{3} x^{\prime}-3 a^{\prime} a^{2} x^{\prime} a-a^{\prime} x^{\prime} a^{3}=0 .
$$

Subtract (20) from (19):

$$
\begin{aligned}
3\left(a a^{\prime}-a^{\prime} a\right) x^{\prime} a^{2}+\left(a^{3} a^{\prime}-a^{\prime} a^{3}\right) x^{\prime}-3\left(a^{2} a^{\prime}-a^{\prime} a^{2}\right) x^{\prime} a & =0 \\
& \text { for all } x, a \in R .
\end{aligned}
$$

Using (17) and (18), we arrive at, after dividing by 3 ,

$$
\left(a a^{\prime}-a^{\prime} a\right)\left(x^{\prime} a^{2}+a^{2} x^{\prime}-2 a x^{\prime} a\right)=0 \quad \text { for all } x, a \in R \text {. }
$$

If $a a^{\prime}-a^{\prime} a \neq 0$ for some $a$, then for that $a$, and all $x$,

$$
x^{\prime} a^{2}+a^{2} x^{\prime}-2 a x^{\prime} a=0 .
$$

Replace $x$ by $a x$ in (21):

$$
a x^{\prime} a^{2}+a^{3} x^{\prime}-2 a^{2} x^{\prime} a+a^{\prime} x a^{2}+a^{2} a^{\prime} x-2 a a^{\prime} x a=0 ;
$$


since

$$
a x^{\prime} a^{2}+a^{3} x^{\prime}-2 a^{2} x^{\prime} a=a\left(x^{\prime} a^{2}+a^{2} x^{\prime}-2 a x^{\prime} a\right)=0
$$

by (21), we have

$$
a^{\prime} x a^{2}+a^{2} a^{\prime} x-2 a a^{\prime} x a=0 \quad \text { for all } x \in R .
$$

Now in (21) replace $x$ by $a: a^{\prime} a^{2}+a^{2} a^{\prime}-2 a a^{\prime} a=0$. Multiply this on the right by $x$.

$$
a^{\prime} a^{2} x+a^{2} a^{\prime} x-2 a a^{\prime} a x=0 \quad \text { for all } x \in R .
$$

Subtract (23) from (22).

$$
a^{\prime}\left(x a^{2}-a^{2} x\right)-2 a a^{\prime}(x a-a x)=0 \quad \text { for all } x \in R .
$$

Replace $x$ by $a x$ in (24).

$$
a^{\prime} a\left(x a^{2}-a^{2} x\right)-2 a a^{\prime} a(x a-a x)=0 \quad \text { for all } x \in R .
$$

Multiply (24) by $a$ on the left.

$$
a a^{\prime}\left(x a^{2}-a^{2} x\right)-2 a^{2} a^{\prime}(x a-a x)=0 \quad \text { for all } x \in R .
$$

Subtract now (25) from (26):

$\left(a a^{\prime}-a^{\prime} a\right)\left(x a^{2}-a^{2} x\right)-2 a\left(a a^{\prime}-a^{\prime} a\right)(x a-a x)=0$ for all $x \in R$.

Since $a a^{\prime}-a^{\prime} a \neq 0$,

(27) $x a^{2}-a^{2} x-2 a(x a-a x)=0 \quad$ for all $x \in R$ if $a a^{\prime}-a^{\prime} a \neq 0$.

So $x a^{2}+a^{2} x-2 a x a=0, a(a x-x a)=(a x-x a) a, I_{a}^{2}=0$. That is, $a$ is central by Theorem 1 or else $a a^{\prime}=a^{\prime} a$, if $R$ is of characteristic different from 2. So when $R$ is of characteristic not $2, a a^{\prime}=a^{\prime} a$ for all $a \in R$; Lemma 3 now finishes the proof. Let $R$ finally be of characteristic 2 . (27) says $a a^{\prime}=a^{\prime} a$ or else $a^{2}$ is central, for all $a \in R$. If $a a^{\prime} \neq a^{\prime} a$ for some $a \in R, a^{2}$ is central and not zero. For if $a^{2}=0$ then $\left(a^{2}\right)^{\prime}$ $=a a^{\prime}+a^{\prime} a=0, a a^{\prime}=a^{\prime} a$. Then $a$ is not a divisor of zero, since if $y a=0, y a^{2}=0, y=0$. Let $x \in R$; we shall prove that $a a^{\prime}$ commutes with $x^{2}$. Either $(a x a)^{2}$ is central, or $(a x a)(a x a)^{\prime}=(a x a)^{\prime}(a x a)$. If $(a x a)^{2}$ is central, $a x a^{2} x a$ is in the center of $R$. Then $a x^{2} a$ is in the center of $R$, since $a^{2}$ is; call it $c$. Then $a c a=a^{2} c$ is in the center of $R$, and equals $a^{2} x^{2} a^{2}$. So $a^{2} x^{2} a^{2}$ is in the center of $R$, and so is $x^{2}$, whence $x^{2}$ commutes with $a a^{\prime}$ if $(a x a)^{2}$ is central. On the other hand, if $x^{2}$ is not central, then $x x^{\prime}=x^{\prime} x$ and $(a x a)(a x a)^{\prime}=(a x a)^{\prime}(a x a)$. Then $(a x a)$ $\cdot\left(a^{\prime} x a+a x^{\prime} a+a x a^{\prime}\right)=\left(a^{\prime} x a+a x^{\prime} a+a x a^{\prime}\right) a x a$, or

$$
a x a a^{\prime} x a+a x a^{2} x^{\prime} a+a x a^{2} x a^{\prime}=a^{\prime} x a^{2} x a+a x^{\prime} a^{2} x a+a x a^{\prime} a x a .
$$


Now $a^{2}$ is central, whence

$$
a x\left(a a^{\prime}+a^{\prime} a\right) x a+\left(a\left(x x^{\prime}+x^{\prime} x\right) a+a x^{2} a^{\prime}+a^{\prime} x^{2} a\right) a^{2}=0 .
$$

But $x x^{\prime}+x^{\prime} x=0$, and $a a^{\prime}+a^{\prime} a$ is central so that

$$
\left(a a^{\prime}+a^{\prime} a\right) a x^{2} a+\left(a x^{2} a^{\prime}+a^{\prime} x^{2} a\right) a^{2}=0 .
$$

Since $a$ is not a right zero divisor,

$$
\begin{aligned}
& \left(a a^{\prime}+a^{\prime} a\right) a x^{2}+\left(a x^{2} a^{\prime}+a^{\prime} x^{2} a\right) a=0, \\
& a x^{2}\left(a a^{\prime}+a^{\prime} a\right)+\left(a x^{2} a^{\prime}+a^{\prime} x^{2} a\right) a=0, \\
& a x^{2} a a^{\prime}+a x^{2} a^{\prime} a+a x^{2} a^{\prime} a+a^{\prime} x^{2} a^{2}=0 .
\end{aligned}
$$

Thus $a x^{2} a a^{\prime}+a^{\prime} x^{2} a^{2}=0 ; a^{2}$ is central so $a x^{2} a a^{\prime}+a^{2} a^{\prime} x^{2}=0 ; a$ is not a left divisor of zero so $x^{2} a a^{\prime}+a a^{\prime} x^{2}=0$, for any $x$ such that $x^{2}$ is not central, hence, for all $x \in R$, as promised; otherwise $a a^{\prime}=a^{\prime} a$. Recourse to the latter part of Lemma 3 shows $a^{3}$ central and $a a^{\prime}$ central or else $a a^{\prime}=a^{\prime} a$. But in the former case, $a \cdot a a^{\prime}=a a^{\prime} \cdot a$; since $a$ is not a zero divisor, $a a^{\prime}=a^{\prime} a$, for all $a \in R$. Lemma 3 completes the proof.

The University of Chicago and

Bell Telephone Laboratories, New York 